\title{
Preferences and behavior of cattle grazing 8 varieties of grasses
}

\author{
DAVID GANSKOPP, BILL MYERS, SCOTT LAMBERT, AND RUBEN CRUZ
}

Authors are rangeland scientist, USDA, Agricultural Research Service, Eastern Oregon Agricultural Research Center, HC 71 4.51 Hwy. 205, Burns, Ore. 97720; Natural Resources Conservation Service (retired), P.O. Box 848, Hines, Ore. 97738; plant resources specialist, Natural Resources Conservation Service, 127 Johnson Hall, NATRS, Washington State Univ., Pullman, Wash. 99164; and graduate research assistant, Department of Rangeland Resources, Oregon State University. Corvallis, Ore. 97331.

\begin{abstract}
We compared the forage preferences of steers grazing among 8 varieties of grasses at 2 stages of phenology on the Northern Great Basin Experimental Range near Burns, Ore. Varieties included: 'Nordan' (Agropyron desertorum (Fischer ex Link)Schultes) and 'CD-II' (A. desertorum X A. cristatum (L.) Gaertner) crested wheatgrass; 'Magnar' and 'Trailhead' Basin wildryes (Leymus cinereus (Scribner \& Merrill) A. Löve); 'Goldar' bluebunch wheatgrass (Pseudoroegneria spicata (Pursh)A. Lõve); 'Bozoisky-Select' Russian wildrye (Psathyrostachys junceus (Fischer) Nevski); 'Bannock' thickspike wheatgrass (Elymus lanceolatus ssp. lanceolatus (Scribner \& J.G. Smith) Gould), and 'Secar' Snake River wheatgrass (proposed nomenclature Elymus lanceolatus ssp. wawawaiensis (Scribner \& J.G. Smith) Gould). Three esophageal-fistulated steers grazed each paddock, with 3 paddocks grazed at the boot stage of development, and 3 paddocks grazed after grasses entered quiescence. In boot-stage trials, steers were very selective and collectively harvested $53 \%$ of total bites from the preferred CD-II and Nordan. These crested wheatgrasses also ranked higher $(P<0.05)$ in bites/visit and time/visit. Magnar, Trailhead, and BozoiskySelect were avoided. When grasses were quiescent, steers were less selective; and CD-II, Nordan, Goldar, Bannock, and Bozoisky-Select were all equally acceptable. Magnar and Trailhead were again avoided. Steers consistently took more bites $(P<0.05)$ from preferred forages and regrazed preferred plants before any variety was depleted. Mean distance traveled between successive feeding stations was greater during bootstage trials $(2.4 \mathrm{~m})$ than at quiescence $(1.4 \mathrm{~m})$, suggesting steers searched among the nearest $\mathbf{4 8}$ neighboring plants in boot-stage trials and the nearest 24 neighbors during quiescence. Measures of grazing time per variety were strongly correlated $(r>0.95$, $P<0.01$ ) with total bites harvested from varieties and are probably adequate for ranking relative preferences of steers. By selectively grazing at both stages of phenology, cattle diets were higher in CP, $P$, and ADL than the standing crop. During boot-stage trials, diets were also higher in $\mathrm{Ca}$ and $\mathrm{Mg}$ than forage analyses would suggest. Except for phosphorus, the nutritive content of all varieties was satisfactory for lactating beef cattle at both stages
\end{abstract}

Eastern Oregon Agr. Res. Ctr., including the Burns and Union stations, is jointly operated by the Oregon Agr. Exp. Sta. of Oregon State Univ. and the USDA-ARS.

Technical Paper No. 10984. Oregon Agricultural Experiment Station

Manuscript accepted 8 Dec. 1996. of phenology. Given their proven ease of establishment, competitive ability, nutritional value, grazing tolerance, and high relative palatability, we suggest the crested wheatgrasses (CD-II and Nordan), are excellent candidates for reclaiming or establishment of pastures for beef production programs in the northern Great Basin.

Key Words: selectivity, palatability, foraging theory, patchy environment, basin wildrye, bluebunch wheatgrass, thickspike wheatgrass, crested wheatgrass, Russian wildrye, Snake River wheatgrass

\section{Resúmen}

Las preferencias forrajeras de novillos pastoreando entre 8 variedades de pastos fueron comparadas durante dos etapas fenológicas en el "Northern Great Basin Experimental Range" cerca de Burns, Ore. En el estudio se incluyeron las siguientes variedades: 'Nordan' (Agropyron desertorum (Fischer ex Link) Schultes) y 'CD-II' (A. desertorum X A. cristatum (L.) Gaertner) de crested wheatgrass; 'Magnar' $y$ 'Trailhead' de Basin wildrye (Leymus cinereus (Scribner \& Merrill) A. Löve); 'Goldar' de bluebunch wheatgrass (Pseudoroegneria spicata (Pursh)A. Löve); 'Bozoisky-Select' de Russian wildrye (Psathyrostachys junceus (Fischer) Nevski); 'Bannock' de thickspike wheatgrass (Elymus lanceolatus ssp. lanceolatus (Scribner \& J.G. Smith) Gould), y 'Secar' de Snake River wheatgrass (nomenclatura propuesta Elymus lanceolatus ssp. wawawaiensis (Scribner \& J.G. Smith) Gould).

Tres novillos con fistulas de esófago pastorearon cada parcela, 3 parcelas fueron pastoreadas durante la fase de encañado y 3 parcelas después de que los pastos entraron en dormancia. Durante las pruebas en la fase de encañado los novillos fueron muy selectivos y, en forma colectiva, consumieron $53 \%$ del total de las mordidas de las variedades preferidas CD-II y Nordan. Estas variedades de crested wheatgrass también obtuvieron altas clasificaciones $(P<0.05)$ en mordidas por visita $y$ tiempo por visita. Las variedades Magnar, Trailhead, y Bozoisky-Select fueron rehusadas. Cuando los pastos estaban en dormancia los novillos fueron menos selectivos y CD-II, Nordan, Goldar, Bannock, y Bozoisky-Select fueron aceptados igualmente. Magnar y Trailhead volvieron a ser rehusados. Consistentemente, los novillos tomaron mas mordidas $(P<0.05)$ de los forrajes preferidos y repastorearon las plantas preferidas antes de que 
ninguna variedad fuera consumida en su totalidad. La distancia media viajada entre estaciones de pastoreo sucesivas fue mayor durante las pruebas de la fase de encañado $(2.4 \mathrm{~m})$ que durante la dormancia $(1.4 \mathrm{~m})$, lo que sugiere que los novillos buscaron entre las 48 plantas mas cercanas durante la fase de encañado y entre las $\mathbf{2 4}$ mas cercanas durante la dormancia. Las medidas de tiempo pastoreando cada variedad fueron altamente correlacionadas $(r>0.95, P<0.01)$ con las mordidas totales tomadas de cada variedad y probablemente sean un indicador adecuado para clasificar la preferencia relativa de los novillos. Debido al pastoreo selectivo en las dos etapas fenologicas, las dietas del ganado fueron mas altas en CP, P, y ADL que el forraje ofrecido. Durante las pruebas en la fase de encañado, las dietas también fueron mas altas en $\mathrm{Ca}$ y $\mathrm{Mg}$ que lo que sugerían los análisis de los forrajes. Con la excepción del $P$, el contenido nutritivo de todas las variedades, en ambas etapas fenologicas fue satisfactorio para ganado de carne en lactancia. Dada la facilidad de establecimiento, habilidad competitiva, valor nutritivo, tolerancia al pastoreo, y alta palatabilidad relativa, sugerimos las variedades de crested wheatgrass (CD-II y Nordan) como excelentes candidatas para reclamación y establecimiento de praderas para producción de carne en la región noroeste del "Great Basin".

Selective foraging by herbivores often affects the character and composition of rangelands (McNaughton and Geordiadis 1986, Crawley 1990, Brown and Stuth 1993, Taylor et al. 1993). Seasonal shifts in forage palatability can be exploited to reduce herbivory in tree plantations (Sharrow et al. 1989), control weeds (Sharrow and Mosher 1982, Walker et al. 1992), and selectively harvest and stimulate growth and quality of forages on rangelands (Anderson and Scherzinger 1975, Reiner and Urness 1982, Gordon 1988).

Because effective techniques for predicting selective grazing by livestock have not been developed (Jones et al. 1994), range managers and researchers must still rely on demonstrated preferences of animals to project how specific forages or mixtures of forages might be accepted (Hart and Hanson 1990). With selections of forages continually being released, it is particularly difficult for managers to evaluate the relative acceptability of new varieties to livestock before committing extensive resources to pasture development or reclamation. Therefore, our objectives were to: 1) determine the relative preferences of steers for 8 varieties of grasses adapted to and available for reclamation of sagebrushsteppe and Great Basin rangelands; 2) describe grazing behavior of steers foraging among grasses of differing palatabilities; and 3) develop a model to consistently predict diet selection of steers from physical and chemical attributes of grasses. Sampling occurred at 2 stages of phenology (boot stage and quiescent) in 1992. Indices of selectivity were related to 8 measures of physical characteristics and 12 chemical constituents of the grasses to determine if relative preferences could be consistently predicted with any combination of these variables.

\section{Materials and Methods}

\section{Pasture Design}

Seed of the 8 varieties were planted and grown in $4 \times 21-\mathrm{cm}$ tubes filled with commercial-potting soil in a greenhouse during the winter and transplanted to paddocks on the Northern Great
Basin Experimental Range $\left(119^{\circ} 43^{\prime} \mathrm{W}, 43^{\circ} 29^{\prime} \mathrm{N}\right)$ in April 1990. Planting design was a randomized complete block in 6 paddocks with each paddock being $20.6 \mathrm{~m}$ to a side. Within each paddock 98 plants of each variety were planted for a total of 784 plants arranged on $76 \mathrm{~cm}$ centers. The position of each plant was randomized in a $28 \times 28$ matrix, so animals could not simply forage along a row or column supporting a single variety. Three additional paddocks were also established and used to precondition animals to forages and familiarize staff and animals with sampling procedures. Soil in the area was a complex of loam and loamy fine sands (Milican coarse-loamy, mixed, frigid Orthidic Durixerolls and Holtle coarse-loamy, mixed, frigid Aridic Duric Haploxerolls, respectively) (Lentz and Simonson 1986). In 1990 the region received $67 \%$ of mean crop-year precipitation (Sneva 1982). Paddocks were irrigated twice during the first growing season to assure establishment, received no supplemental water thereafter, and weeds were hoed as needed. Paddocks were also mowed each fall to eliminate standing litter.

The 8 varieties included 2 crested wheatgrasses, 'Nordan' (Agropyron desertorum (Fischer ex Link)Schultes) and 'CD-II' a crossed product of $A$. desertorum and A. cristatum (L.)Gaertner; 2 Basin wildryes, 'Magnar' and 'Trailhead' (Leymus cinereus (Scribner \& Merrill) A. Löve); 'Goldar', bluebunch wheatgrass (Pseudoroegneria spicata (Pursh)A. Löve); 'Bozoisky-Select' Russian wildrye (Psathyrostachys junceus (Fischer) Nevski); 'Bannock' thickspike wheatgrass (Elymus lanceolatus (Scribner \& J.G. Smith) Gould); and 'Secar' Snake River wheatgrass (proposed nomenclature Elymus lanceolatus ssp. wawawaiensis (Scribner \& J.G. Smith) Gould). CD-II seed was provided by USDA-ARS, Logan, Ut. Magnar, Goldar, and Bannock were products of the Aberdeen, Ida. Plant Materials Center. Trailhead and Bozoisky-Select were received from the Bridger, Mont. Plant Materials Center. Secar came from the Pullman, Wash. Plant Materials Center, and Nordan seed was acquired commercially.

\section{Plant Sampling}

Before a paddock was grazed we measured the basal area and greatest culm height of 5 randomly selected plants of each variety. Basal area was derived from a plant's maximum diameter, a second diameter perpendicular to the first, and solving for the area of an ellipse (Jones et al. 1994). This was followed by clipping to a $2.5-\mathrm{cm}$ stubble to obtain green and oven-dry $\left(60^{\circ} \mathrm{C}\right)$ biomass and a measure of leaf:stem ratio. Samples were then composited by variety within a paddock, and retained for grinding (20 mesh screen) and later chemical analyses.

Variables derived from these data or plant materials included basal area $\left(\mathrm{cm}^{2}\right)$, maximum plant height $(\mathrm{cm})$, biomass $(\mathrm{g})$, as fed moisture content $(\%)$, forage density (biomass $/ \mathrm{cm}^{2}$ basal area), forage bulk density (biomass/liter- an integration of biomass, basal area, and height), leaf:stem ratio, and exterior culm or stem angle (degrees from horizontal). Chemical analyses included crude protein (CP) (AOAC 1980), invitro organic matter disappearance (IVOMD) (Tilley and Terry 1963), acid detergent fiber (ADF), and acid detergent lignin (ADL) (Goering and Van Soest 1970), indigestible acid detergent fiber (Galyean et al. 1987), and phosphorus $(\mathrm{P})$, potassium $(\mathrm{K})$, magnesium $(\mathrm{Mg})$, and calcium (Ca) by inductive coupled plasma techniques at the Research Extension Analytical Laboratory of Ohio State University.

The day after a paddock was grazed, a single technician categorically scored utilization of each plant $(n=784)$ with indices 
indicating $(0)=$ no utilization, $(1)=1-20 \%$ of plant weight removed, $(2)=21-40 \%$ removed, and $(3)=40+\%$ removed. Variables derived from these data included mean utilization (expressed as a percentage of all 98 plants of each variety), number of plants grazed, and mean utilization of the grazed plants with a variable sample size.

\section{Grazing Behavior Sampling and Analyses}

To provide a measure of seasonal variation in palatability, trials were conducted during 2 stages of phenology (boot stage and quiescent). Three paddocks were grazed when forages were green and growing (20-23 May) and 3 grazed when the grasses were quiescent (19-21 Aug., 1992). During the May sampling Basin wildryes were in the vegetative stage while the remaining 6 varieties were in the boot stage of development. In August all herbage was cured and seed heads were shattering. Two days before trials, the 5 esophageal-fistulated steers were placed in the 3 extra paddocks to allow familiarization with the forages. Previously they were maintained on bluebunch wheatgrass/Idaho fescue (Festuca idahoensis Elmer) native range.

Rows and columns of plants in the paddocks were numbered and marked, so a coordinate system could be used to identify each plant. Each paddock was grazed by 3 randomly chosen steers. To inhibit social facilitation each animal foraged alone. Each steer was allowed to visit 83 plants before it exited the paddock. Visits included only plants actually grazed, and subsequent returns to and regrazing of individual plants were scored as additional visits. This protocol allowed sustained selective opportunity for the steers because ungrazed plants of each variety were still available when trials ended. As each steer grazed, it was accompanied by an observer equipped with a backpack mounted platform and lap-top computer. The observer tallied each bite as material was severed from a plant and noted when the animal abandoned a feeding station and began walking in search of another. Steers were quite tame and would tolerate observers within 2 to $5 \mathrm{~m}$. Because some varieties could not be distinguished by cursory examination, 2 additional technicians moved along the $\mathrm{X}$ and $\mathrm{Y}$ axes of the enclosing fence and simultaneously recorded row/column coordinates of each grazed plant. Voice communication was used to coordinate data acquisition among technicians, and all judgement calls were decided by the computer equipped individual.

All data were tallied by variety across steers within a paddock. Feeding station coordinates, maps of the paddocks, and bitecount data were integrated to yield 10 variables for each variety: visits (the number of times a variety was grazed including repeat visits), total bites (the sum of all bites taken from a variety), percentage of single-bite visits (proportion of visits to a variety in which steers harvested a single bite $\times 100$ ), mean bites per visit for each variety, total time expended on each variety (derived by difference between the time of the first and last bite at each feeding station), mean time per visit for each variety, mean bite rate (bites/minute derived from number of bites and duration of visit at each feeding station), number of plants grazed (the total number of plants of a variety that were foraged upon), number of plants regrazed (the number of plants foraged upon 2 or more times), and mean distance between feeding stations (determined from the coordinate data). Mean distance was probably underestimated in these trials, because we assumed straight-line travel between feeding stations.

\section{Statistical Analyses}

With the exception of visits, all plant and animal variables were analyzed with a split-plot analysis of variance with 3 replications. Because stages of phenology or growth can not be randomized in the field, the 2 stages of phenology served as whole-plots and the 8 varieties as sub-plots. Replication $\times$ phenology $(2 \mathrm{df})$ was the error term for the phenology effect ( $1 \mathrm{df}$ ), and the phenology $x$ replication $X$ variety ( $28 \mathrm{df}$ ) error term was used to test for variety ( $7 \mathrm{df})$ and variety $\times$ phenology effects $(7 \mathrm{df})$. Mean separations were accomplished with Fisher's protected LSD procedures, and statistical significance for all tests was assumed at $P<0.05$. Degree of agreement in rankings of varieties by several variables was quantified with Spearman's rank correlation coefficient $\left(r_{s}\right)$.

A Pearson's correlation matrix was assembled to explore relationships among the 13 grazing behavior variables and the 8 physical and 9 chemical attributes of the forages at each stage of phenology. Correlation coefficients were deemed statistically significant at $\mathrm{P}<0.01$, and we arbitrarily decided that correlations must equal or exceed $0.95\left(r^{2} \geq 0.90\right.$ to be of predictive value. Step-wise regression analyses were used to detect potentially consistent predictors of selection at both stages of phenology by relating total bites removed from each variety with their 8 physical and 9 chemical characteristics. Variable entry into the models was assumed significant at $\mathbf{P}<0.05$. For all regression analyses $n$ for each variable equaled 24 ( 3 replications $\times 8$ varieties).

\section{Results and Discussion}

\section{Total Bites}

The phenology main effect or phenology $x$ variety interaction was significant for 25 of 29 variables analyzed, so data are presented separately for the boot and quiescent stages of phenology (Table 1). Steers foraged selectively during both stages of phenology but were more focused during boot-stage trials when over $50 \%$ of their total bites were removed from only 2 varieties (CDII and Nordan). Steers harvested more total bites $(2,128)$ from each paddock during boot-stage trials than when the grasses were dormant $(1,216)$. For total bites they exhibited a 23 -fold range among variety means during boot-stage trials and only a 10-fold range when grasses were quiescent.

During boot-stage trials forage rankings could be separated into 3 distinct groups. CD-II was clearly the most preferred forage with $31 \%$ of total bites. Successively ranked as acceptable forages were Nordan, Goldar, Bannock, and Secar, with Nordan scoring higher $(P<0.05)$ than Bannock or Secar. Scoring significantly lower in acceptability were Bozoisky-Select, Trailhead, and Magnar which collectively accounted for less than $6 \%$ of total bites harvested.

When grasses were quiescent, steers were less selective, and forage rankings could only be separated into 2 distinct categories (Table 1). Five varieties (CD-II, Nordan, Bannock Goldar, and Bozoisky-Select) were considered acceptable. Magnar and Trailhead were avoided and collectively contributed less than $5 \%$ to the total bites harvested. Secar, although poorly accepted by the steers, was given an intermediate ranking; because it could not be excluded from either grouping.

Jones et al. (1994) reported heifers preferred 'Hycrest' and Nordan crested wheatgrasses to several varieties of thickspike and bluebunch wheatgrass during the boot-stage of phenology. Sheep, Angora goats, Spanish goats, and black-tailed jackrabbits 
Table 1. Means ( $\pm S E n=3$ ) of 9 variables describing selective grazing behavior of steers foraging on 8 varieties of grasses at 2 stages of phenology on the Northern Great Basin Experimental Range near Burns, Ore.

\begin{tabular}{|c|c|c|c|c|c|c|c|c|c|}
\hline & Visits & $\begin{array}{l}\text { Total } \\
\text { bites }\end{array}$ & $\begin{array}{l}\text { Bites } \\
\text { per } \\
\text { visit }\end{array}$ & $\begin{array}{c}\text { Single } \\
\text { bite } \\
\text { visits }\end{array}$ & $\begin{array}{l}\text { Total } \\
\text { time }\end{array}$ & $\begin{array}{l}\text { Time } \\
\text { per } \\
\text { visit }\end{array}$ & $\begin{array}{c}\text { Bites } \\
\text { per } \\
\text { minute }\end{array}$ & $\begin{array}{l}\text { Plants } \\
\text { grazed }\end{array}$ & $\begin{array}{l}\text { Plants } \\
\text { regrazed }\end{array}$ \\
\hline & & & & $(\%)$ & (sec) & (sec) & & (no) & (no) \\
\hline Yariety & \multicolumn{9}{|c|}{ Boot Stage } \\
\hline CD-П & $57.0 \pm 6.7$ & $661 \pm 141.6 d^{1}$ & $11.3 \pm 1.1 \mathrm{~d}$ & $11.7 \pm 3.2 \mathrm{a}$ & $1097 \pm 327 \mathrm{e}$ & $18.4 \pm 3.3 \mathrm{e}$ & $38.0 \pm 3.0 \mathrm{a}$ & $40 \pm 1.2 b$ & $13 \mathrm{c}$ \\
\hline Nordan & $41.0 \pm 4.6$ & $469 \pm 119.9 \mathrm{c}$ & $11.4 \pm 2.2 \mathrm{~d}$ & $17.9 \pm 6.0 \mathrm{a}$ & $805 \pm 284 d$ & $19.3 \pm 5.4 \mathrm{e}$ & $37.3 \pm 3.5 a$ & $31 \pm 2.0 \mathrm{~b}$ & $8 \mathrm{~b}$ \\
\hline Goldar & $47.0 \pm 5.5$ & $348 \pm 34.8 b c$ & $7.5 \pm 0.6 c$ & $18.9 \pm 3.5 \mathrm{a}$ & $553 \pm 56 \mathrm{~cd}$ & $12.1 \pm 1.8 \mathrm{~d}$ & $37.9 \pm 2.6 \mathrm{a}$ & $35 \pm 3.7 b$ & $10 b c$ \\
\hline Secar & $40.0 \pm 2.1$ & $260 \pm 73.1 b$ & $6.4 \pm 1.5 b c$ & $21.6 \pm 6.3 \mathrm{a}$ & $337 \pm 106 a b c$ & $8.3 \pm 2.3 \mathrm{bcd}$ & $47.2 \pm 2.1 \mathrm{ab}$ & $31 \pm 1.0 \mathrm{~b}$ & $7 \mathrm{~b}$ \\
\hline Bannock & $36.7 \pm 2.8$ & $274 \pm 11.3 b$ & $7.5 \pm 0.3 \mathrm{c}$ & $26.3 \pm 1.0 \mathrm{ab}$ & $387 \pm 15$ bc & $10.7 \pm 1.2 \mathrm{~cd}$ & $42.7 \pm 3.0 \mathrm{ab}$ & $31 \pm 1.9 b$ & $5 a b$ \\
\hline Bozoisky-Select & $9.0 \pm 2.6$ & $49 \pm 26.5 a$ & $4.6 \pm 1.4 \mathrm{ab}$ & $39.8 \pm 20.4 b$ & $70 \pm 35 \mathrm{ab}$ & $6.6 \pm 2.0 \mathrm{abc}$ & $42.7 \pm 4.0 \mathrm{ab}$ & $8 \pm 1.7 a$ & la \\
\hline Magnar & $9.0 \pm 2.6$ & $28 \pm 12.8 \mathrm{a}$ & $2.9 \pm 0.5 \mathrm{a}$ & $39.5 \pm 6.2$ b & $27 \pm 12$ a & $2.7 \pm 0.7 \mathrm{a}$ & $67.9 \pm 10.4 \mathrm{c}$ & $8 \pm 2.0 \mathrm{a}$ & la \\
\hline Trailhead & $9.3 \pm 1.5$ & $42 \pm 16.6 \mathrm{a}$ & $4.2 \pm 1.1 \mathrm{ab}$ & $36.8 \pm 17.4 \mathrm{~b}$ & $49 \pm 20 a$ & $4.8 \pm 1.3 \mathrm{ab}$ & $52.9 \pm 4.4 \mathrm{~b}$ & $7 \pm 0.9 a$ & $2 a$ \\
\hline \multirow[t]{2}{*}{ Mcan } & 31.1 & $266 A$ & $7.0 \mathrm{~A}$ & $26.6 \mathrm{~A}$ & $415 A$ & $10.4 \mathrm{~A}$ & $45.8 \mathrm{~A}$ & $24 \mathrm{~A}$ & $6 \mathrm{~A}$ \\
\hline & \multicolumn{9}{|c|}{ Quiescent } \\
\hline CD-II & $54.3 \pm 4.4$ & $293 \pm 36.9 c$ & $5.4 \pm 0.5 b$ & $13.1 \pm 1.6 \mathrm{ab}$ & $391 \pm 59$ b & $7.2 \pm 1.1 \mathrm{ab}$ & $45.5 \pm 2.8 \mathrm{a}$ & $40 \pm 4.0 \mathrm{~d}$ & $12 d$ \\
\hline Nordan & $49.7 \pm 9.3$ & $261 \pm 44.8 c$ & $5.3 \pm 0.2 b$ & $10.2 \pm 1.8 \mathrm{a}$ & $357 \pm 41 \mathrm{ab}$ & $7.4 \pm 0.7 \mathrm{~b}$ & $43.4 \pm 3.2 \mathrm{a}$ & $38 \pm 6.4 \mathrm{~cd}$ & $9 \mathrm{~cd}$ \\
\hline Goldar & $37.7 \pm 7.9$ & $179 \pm 57.1 \mathrm{abc}$ & $4.5 \pm 0.7 \mathrm{ab}$ & $14.1 \pm 4.0 \mathrm{ab}$ & $183 \pm 61 a b$ & $4.5 \pm 0.8 \mathrm{ab}$ & $59.6 \pm 1.3 b c$ & $30 \pm 6.3 \mathrm{bcd}$ & $7 \mathrm{bc}$ \\
\hline Secar & $26.0 \pm 6.1$ & $69 \pm 30.0 \mathrm{ab}$ & $2.5 \pm 0.5 \mathrm{a}$ & $38.7 \pm 6.2 \mathrm{c}$ & $71 \pm 38 a b$ & $2.3 \pm 0.7 \mathrm{a}$ & $65.3 \pm 6.5 \mathrm{c}$ & $21 \pm 3.5 b$ & $4 \mathbf{a b}$ \\
\hline Bannock & $36.3 \pm 2.7$ & $198 \pm 29.8 b c$ & $5.4 \pm 0.5 \mathrm{~b}$ & $12.7 \pm 5.0 \mathrm{ab}$ & $276 \pm 48 \mathrm{ab}$ & $7.5 \pm 0.8 \mathrm{~b}$ & $43.7 \pm 1.6 \mathrm{a}$ & $28 \pm 2.3 \mathrm{bc}$ & $7 \mathrm{bc}$ \\
\hline Bozoisky-Select & $29.3 \pm 9.1$ & $156 \pm 49.7 a b c$ & $5.3 \pm 0.5 b$ & $20.4 \pm 2.9 \mathrm{ab}$ & $246 \pm 90 \mathrm{ab}$ & $7.8 \pm 1.4 \mathrm{~b}$ & $42.1 \pm 5.3 \mathrm{a}$ & $25 \pm 6.6 b$ & $4 a b$ \\
\hline Magnar & $9.0 \pm 2.0$ & $28 \pm 5.7 \mathrm{a}$ & $3.2 \pm 0.2 \mathrm{ab}$ & $37.6 \pm 5.4 \mathrm{c}$ & $37 \pm 8 a$ & $4.1 \pm 0.2 \mathrm{ab}$ & $46.1 \pm 1.3 \mathrm{a}$ & $8 \pm 2.0 \mathrm{a}$ & $1 \mathrm{a}$ \\
\hline Trailhead & $6.7 \pm 2.7$ & $28 \pm 11.4 \mathrm{a}$ & $4.2 \pm 0.1 \mathrm{ab}$ & $26.1 \pm 3.9 b c$ & $33 \pm 14 a$ & $4.9 \pm 0.4 \mathrm{ab}$ & $51.7 \pm 3.2 \mathrm{ab}$ & $6 \pm 2.4 a$ & $0 \mathrm{a}$ \\
\hline Mean & 31.1 & $152 \mathrm{~B}$ & $4.5 \mathrm{~A}$ & $21.6 \mathrm{~A}$ & $199 \mathrm{~A}$ & $5.7 \mathrm{~A}$ & $49.7 \mathrm{~A}$ & $25 \mathrm{~A}$ & $5 \mathrm{~A}$ \\
\hline
\end{tabular}

${ }^{1}$ Variety means within a column and stage of phenology sharing a common lower case letter are not significantly different $(P>0.05)$.

Means in columns sharing a common upper case letter are not significantly different $(\mathrm{P}>0.05)$.

have also exhibited strong preferences for crested wheatgrass (Shewmaker et al. 1989, Ganskopp et al. 1993, and 1996, and Fajemisin et al. 1996).

\section{Bites Per Visit}

Bites per visit spanned a 4-fold range at the boot stage $(\bar{x}=7.0)$ and only a 2 -fold range during quiescence $(\bar{x}=4.5)$, but there was no difference in bites/visit between the 2 stages of phenology. Differences among varieties were greatest during the boot stage when steers averaged 11 bites per visit from the 2 crested wheatgrasses and 2.9 bites from each Magnar plant. Separations among varieties were less prominent at quiescence, but a difference $(P<0.05)$ was noted between the crested wheatgrasses and the less preferred Secar.

In trials like these, variability in bites per visit may at times be due to differences in production among varieties, especially if forage availability is greatly reduced as the trials progress. Our measures of plant biomass (Table 2) did not support this. Although plant biomass increased $(P<0.05)$ between the 2 sampling periods, e.g., from 16.5 to $53.9 \mathrm{~g}$ plant $^{-1}$, no significant differences in biomass occurred among varieties within either stage of phenology. Consequently, we detected no significant correlations $(P>0.01)$ between plant biomass and bites per visit at either stage of phenology.

Many visits by steers to less preferred varieties ended with removal of only 1 bite (Table 1). This behavior was most prominent during our boot-stage sampling when the probability of a single-bite visit averaged $38 \%$ for the less preferred BozoiskySelect, Magnar, and Trailhead and only $19 \%$ among the other 5 varieties. Again, there were no significant correlations between single-bite visits and plant biomass.

\section{Grazing Time}

Total time grazing each variety (Table 1) strongly correlated with total bites. Relative rankings among varieties with the 2 variables were identical $\left(r_{s}=1.0\right)$ during our boot-stage trials and nearly identical $\left(r_{s}=0.97\right)$ when forages were quiescent. The extremely strong correlations ( $r=0.97$ boot-stage and 0.99 quiescence) between total time and total bites suggests that less demanding measures of foraging time, where observers simply input start and stop times at each feeding station instead of tallying individual bites, would be adequate for ranking forage preferences.

Mean time per visit was $10 \mathrm{sec}$ in boot-stage trials and $6 \mathrm{sec}$ $(\mathrm{P}=0.11)$ when grasses were dormant (Table 1$)$. During bootstage trials, steers spent about $18.8 \mathrm{sec}$ at crested wheatgrass plants and roughly 3 to $7 \mathrm{sec}$ with the less preferred BozoiskySelect, Magnar, and Trailhead varieties. At quiescence, the only clear separation among varieties involved the less preferred Secar and the more readily accepted Bannock, Bozoisky-Select, and Nordan varieties. Once again, one might expect a relationship between time per visit and plant biomass, but correlation coefficients were only 0.44 and 0.13 for boot stage and quiescent analyses, respectively. Although time per visit and total bite rankings of varieties were identical in boot-stage trials $\left(r_{s}=1.0\right)$ Spearman's rank correlation coefficient declined to 0.40 for rankings by the 2 variables at quiescence.

\section{Number of Plants Grazed}

Results from the number of plants grazed (Table 1) parallel those obtained with total bites and total time. Only 40 of 98 total plants were grazed for even the most frequently utilized varieties, suggesting selective opportunities were not greatly diminished as 
Table 2. Physical characteristies ( \pm SE) of 8 varieties of grasses grazed by steers in preferences trials at 2 stages of phenology on the Northern Great Basin Experimemtal Range.

\begin{tabular}{|c|c|c|c|c|c|c|c|}
\hline & $\begin{array}{l}\text { Basal } \\
\text { area }\end{array}$ & $\begin{array}{c}\text { Plant } \\
\text { height }\end{array}$ & $\begin{array}{c}\text { Plant } \\
\text { biomass }\end{array}$ & $\begin{array}{l}\text { Moisture } \\
\text { content }\end{array}$ & $\begin{array}{l}\text { Forage } \\
\text { density }\end{array}$ & $\begin{array}{c}\text { Bulk } \\
\text { density }\end{array}$ & $\begin{array}{c}\text { Leaf/stem } \\
\text { ratio }\end{array}$ \\
\hline Variety & $\left(\mathrm{cm}^{2}\right)$ & \multicolumn{5}{|c|}{ Boot Stage } & \\
\hline $\begin{array}{l}\text { CD- II } \\
\text { Nordan } \\
\text { Goldar } \\
\text { Secar } \\
\text { Bannock } \\
\text { Bozoisky-Select } \\
\text { Magnar } \\
\text { Trailhead }\end{array}$ & $\begin{array}{r}164 \pm 27.6 \mathrm{a}^{3} \\
138 \pm 33.5 \mathrm{a} \\
88 \pm 39.3 \mathrm{a} \\
86 \pm 21.9 \mathrm{a} \\
149 \pm 37.4 \mathrm{a} \\
213 \pm 28.4 \mathrm{a} \\
88 \pm 47.3 \mathrm{a} \\
96 \pm 26.8 \mathrm{a}\end{array}$ & $\begin{array}{l}37.7 \pm 3.3 \mathrm{a} \\
38.3 \pm 0.7 \mathrm{a} \\
33.7 \pm 1.8 \mathrm{a} \\
32.3 \pm 4.1 \mathrm{a} \\
35.0 \pm 1.5 \mathrm{a} \\
38.3 \pm 1.8 \mathrm{a} \\
39.7 \pm 4.5 \mathrm{a} \\
45.3 \pm 8.4 \mathrm{a}\end{array}$ & $\begin{array}{l}26.1 \pm 2.4 \mathrm{a} \\
19.7 \pm 5.9 \mathrm{a} \\
15.3 \pm 2.6 \mathrm{a} \\
10.2 \pm 0.2 \mathrm{a} \\
16.2 \pm 3.9 \mathrm{a} \\
16.5 \pm 4.8 \mathrm{a} \\
14.1 \pm 2.1 \mathrm{a} \\
14.2 \pm 3.3 \mathrm{a}\end{array}$ & $\begin{array}{l}69.0 \pm 1.0 \mathrm{abc} \\
70.3 \pm 0.7 \mathrm{bc} \\
66.7 \pm 1.8 \mathrm{a} \\
67.0 \pm 2.0 \mathrm{ab} \\
67.7 \pm 0.7 \mathrm{ab} \\
70.7 \pm 0.3 \mathrm{bc} \\
76.3 \pm 1.2 \mathrm{~d} \\
71.7 \pm 1.5 \mathrm{c}\end{array}$ & $\begin{array}{l}0.17 \pm 0.03 \mathrm{ab} \\
0.17 \pm 0.09 \mathrm{ab} \\
0.23 \pm 0.07 \mathrm{~b} \\
0.14 \pm 0.05 \mathrm{ab} \\
0.11 \pm 0.01 \mathrm{ab} \\
0.08 \pm 0.02 \mathrm{a} \\
0.23 \pm 0.07 \mathrm{~b} \\
0.15 \pm 0.02 \mathrm{ab}\end{array}$ & $\begin{array}{l}4.5 \pm 0.8 \mathrm{a} \\
4.4 \pm 2.2 \mathrm{a} \\
6.9 \pm 2.0 \mathrm{a} \\
4.5 \pm 1.6 \mathrm{a} \\
3.2 \pm 0.4 \mathrm{a} \\
2.0 \pm 0.5 \mathrm{a} \\
6.4 \pm 2.3 \mathrm{a} \\
3.5 \pm 0.4 \mathrm{a}\end{array}$ & $\begin{array}{l}1.4 \pm 0.1 \mathrm{a} \\
3.0 \pm 0.8 \mathrm{ab} \\
3.5 \pm 0.4 \mathrm{bc} \\
2.2 \pm 0.3 \mathrm{ab} \\
2.8 \pm 0.7 \mathrm{abc} \\
1.4 \pm 0.1 \mathrm{a} \\
4.8 \pm 0.9 \mathrm{~d} \\
4.3 \pm 1.6 \mathrm{~cd}\end{array}$ \\
\hline Mean & $128 \mathrm{~B}$ & $37.56 \mathrm{~A}$ & $16.5 \mathrm{~A}$ & \multicolumn{3}{|c|}{ Quiescent } & $2.9 \mathrm{~A}$ \\
\hline $\begin{array}{l}\text { CD-II } \\
\text { Nordan } \\
\text { Goldar } \\
\text { Secar } \\
\text { Bannock } \\
\text { Bozoisky-Select } \\
\text { Magnar } \\
\text { Trailhead }\end{array}$ & $\begin{array}{l}187 \pm 38.5 \mathrm{abc} \\
304 \pm 59.2 \mathrm{~d} \\
190 \pm 24.8 \mathrm{abc} \\
147 \pm 27.2 \mathrm{ab} \\
611 \pm 190.5 \mathrm{~d} \\
266 \pm 33.7 \mathrm{bc} \\
125 \pm \mathrm{a} 25.0 \mathrm{~b} \\
80 \pm \mathrm{a} 19.5\end{array}$ & $\begin{array}{l}40.3 \pm 4.3 \mathrm{a} \\
42.3 \pm 2.3 \mathrm{ab} \\
44.7 \pm 3.8 \mathrm{ab} \\
50.0 \pm 4.0 \mathrm{ab} \\
55.0 \pm 4.6 \mathrm{ab} \\
56.7 \pm 6.7 \mathrm{bc} \\
71.7 \pm 13.2 \mathrm{~cd} \\
81.3 \pm 11.2 \mathrm{~d}\end{array}$ & $\begin{array}{l}42.2 \pm 8.4 b \\
60.7 \pm 17.8 b \\
54.3 \pm 6.8 b \\
53.7 \pm 12.9 b \\
79.4 \pm 14.5 b \\
60.9 \pm 15.1 b \\
45.6 \pm 19.1 b \\
34.5 \pm 7.9 b\end{array}$ & $\begin{array}{l}30.4 \pm 0.5 \mathrm{a} \\
27.7 \pm 0.9 \mathrm{a} \\
23.8 \pm 1.6 \mathrm{a} \\
26.7 \pm 0.9 \mathrm{a} \\
22.7 \pm 3.5 \mathrm{a} \\
24.7 \pm 1.5 \mathrm{a} \\
29.0 \pm 1.8 \mathrm{a} \\
26.2 \pm 3.4 \mathrm{a}\end{array}$ & $\begin{array}{l}0.23 \pm 0.02 \mathrm{abc} \\
0.19 \pm 0.04 \mathrm{ab} \\
0.30 \pm 0.08 \mathrm{bcd} \\
0.36 \pm 0.06 \mathrm{~d} \\
0.15 \pm 0.04 \mathrm{a} \\
0.23 \pm 0.04 \mathrm{abc} \\
0.35 \pm 0.09 \mathrm{~cd} \\
0.44 \pm 0.01 \mathrm{~d}\end{array}$ & $\begin{array}{l}5.9 \pm 1.0 \mathrm{bcd} \\
4.5 \pm 1.7 \mathrm{ab} \\
6.6 \pm 1.1 \mathrm{~cd} \\
7.3 \pm 1.3 \mathrm{~d} \\
2.7 \pm 0.7 \mathrm{a} \\
4.3 \pm 1.1 \mathrm{ab} \\
4.8 \pm 0.6 \mathrm{bc} \\
5.6 \pm 1.0 \mathrm{bcd}\end{array}$ & $\begin{array}{l}0.4 \pm 0.0 \mathrm{a} \\
0.5 \pm 0.2 \mathrm{a} \\
0.8 \pm 0.1 \mathrm{a} \\
0.8 \pm 0.2 \mathrm{a} \\
1.3 \pm 0.3 \mathrm{ab} \\
2.7 \pm 1.1 \mathrm{~b} \\
1.1 \pm 0.1 \mathrm{ab} \\
1.0 \pm 0.2 \mathrm{ab}\end{array}$ \\
\hline Mean & $239 \mathrm{~B}$ & 55.3 B & $53.9 \mathrm{~B}$ & 26.4 B & $0.28 \mathrm{~B}$ & $5.2 \mathrm{~B}$ & $1.1 \mathrm{~B}$ \\
\hline
\end{tabular}

Forage density=plant biomass $/ \mathrm{cm}^{2}$ basal area.

${ }^{2}$ Bulk density $=$ plant biomass/(basal area $\times$ plant height/1000) expressed as grams per liter.

${ }^{3}$ Variety means within a column and stage of phenology sharing a common lower case letter are not significantly different $(P>0.05)$.

Means in columns sharing a common upper case letter are not significantly different $(P>0.05)$.

trials progressed. On average 20 to $25 \%$ of the total visits were returns to previously grazed plants (Table 1). With preferred forages like CD-II, $30-33 \%$ of grazed plants were regrazed, while nearly $60 \%$ of the population remained unscathed. Plants of avoided varieties (Magnar and Trailhead) were less likely to be regrazed $(P<0.05)$ than preferred varieties.

\section{Post-Grazing Utilization Measures}

Estimates of the number of plants utilized in post-grazing examinations of paddocks (Table 3 ) were strongly correlated ( $r=$ $0.91-0.96)$ with our direct observations of plants grazed (Table 1), but consistent discrepancies occurred between the 2 methods. Utilization data (Table 3 ) consistently implied steers used more plants than we noted in our direct observations. Paired t-tests comparing the 2 methods revealed differences $(P<0.05)$ between the 2 measures of 3.5 and 7.8 plants per variety for boot stage and quiescent trials, respectively. We can offer no sound explanations for these differences. Lagomorphs were excluded from paddocks by a $2.5-\mathrm{cm}$ mesh fence and small rodent grazing was not apparent. Other possible causes include insect defoliation, a sub-conscious desire by technicians to detect utilization, and breakage of brittle standing material may have been falsely interpreted as utilization during quiescent trials. Although rankings of forages based on post grazing counts of grazed plants and percent of plant weight utilized (Table 3) closely corresponded with our bite count data (mean $r_{s}=0.90$ ), we agree with others (Laycock et al. 1972, McInnis et al. 1983) that utilization methods are not a preferred means of quantifying herbivore diets.

Mean levels of utilization varied $(P<0.05)$ among varieties at each stage of phenology (Table 3 ) with proportionally more material removed from preferred than avoided varieties. In bootstage trials an 11-fold range occurred between the most heavily utilized Goldar and least-grazed Bozoisky-Select. At quiescence, the extremes (Nordan $=15.2 \%$ and Trailhead $=2.0 \%$ ) displayed only a 7-fold range. Mean utilization is an integration of the number of plants sampled, amount of herbage available, and the proportion of material removed from grazed plants. These values accurately describe the relative amount of material removed from the entire population of plants, but if many zero's occur in the data, they may not accurately characterize the amount of material removed from plants that were actually grazed. Our data clearly illustrated this when utilization means were based solely on grazed plants (Table 3). Although estimates of grazed plant utilization ranged from 2 to 15 times greater than population means, the magnitude of variation among varieties was greatly reduced with approximately a 1.8 and 1.6 fold spread for boot stage and quiescent trials, respectively. Grazed Goldar plants were most heavily utilized in boot-stage trials followed by CD-II, Bannock, and Nordan. Bozoisky-Select and Magnar were least utilized. Grazed plant utilization was more equitable in quiescent trials with no differences among the more heavily utilized CD-II, Nordan, Goldar, Magnar, and Trailhead. Bozoisky-Select was again one of the least utilized varieties.

\section{Physical Characteristics of Varieties}

Physical attributes of the grasses are presented in Table 2. Plant height and basal area were similar in boot-stage trials, but differences $(P<0.05)$ occurred among varieties for both variables after forages reached full stature. At quiescence Trailhead and Magnar were tallest, with only slight differences among the remaining 6 varieties. Significant herbage production occurred between trials, as mean biomass per plant increased from 17 to 54 grams. High variability in biomass of individual plants hindered separations among varieties at both stages of phenology (Table 2). 
Table 3. Number of plants utilized, mean level of utilization (\% of plant weight) for all plants, and mean level of utilization for grazed plants in paddocks supporting 8 varieties of grasses that were selectively grazed by steers at 2 stages of phenology on the Northern Great Basin Experimental Range near Burns, Ore.

\begin{tabular}{|c|c|c|c|}
\hline & $\begin{array}{l}\text { Number } \\
\text { of plants } \\
\text { utilized }\end{array}$ & $\begin{array}{c}\text { Mean } \\
\text { utilization } \\
(\mathrm{n}=98)\end{array}$ & $\begin{array}{c}\text { Grazed plant } \\
\text { utilization } \\
(n=\text { variable) }\end{array}$ \\
\hline Variety & (no) & $\begin{array}{c}(\%) \\
\text { Boot Stage }\end{array}$ & (\%) \\
\hline $\begin{array}{l}\text { CD-II } \\
\text { Nordan } \\
\text { Goldar } \\
\text { Secar } \\
\text { Bannock } \\
\text { Bozoisky-Select } \\
\text { Magnar } \\
\text { Trailhead }\end{array}$ & $\begin{array}{c}46 \pm 2.7 \mathrm{c}^{1} \\
39 \pm 1.8 \mathrm{bc} \\
41 \pm 7.4 \mathrm{bc} \\
34 \pm 4.4 \mathrm{~b} \\
33 \pm 4.2 \mathrm{~b} \\
7 \pm 1.2 \mathrm{a} \\
10 \pm 1.3 \mathrm{a} \\
9 \pm 0.9 \mathrm{a}\end{array}$ & $\begin{array}{c}11.9 \pm 1.0 \mathrm{~cd} \\
9.5 \pm 0.2 \mathrm{bc} \\
13.3 \pm 3.5 \mathrm{~d} \\
7.6 \pm 1.1 \mathrm{~b} \\
8.4 \pm 1.2 \mathrm{bc} \\
1.2 \pm 0.2 \mathrm{a} \\
1.9 \pm 0.2 \mathrm{a} \\
2.1 \pm 0.6 \mathrm{a}\end{array}$ & $\begin{array}{l}25.7 \pm 1.4 \mathrm{~b} \\
24.6 \pm 0.9 \mathrm{~b} \\
31.4 \pm 2.4 \mathrm{c} \\
22.4 \pm 0.7 \mathrm{ab} \\
25.3 \pm 1.8 \mathrm{~b} \\
17.8 \pm 0.6 \mathrm{a} \\
19.9 \pm 0.8 \mathrm{a} \\
21.3 \pm 4.6 \mathrm{ab}\end{array}$ \\
\hline Mean & $27 \mathrm{~A}$ & $\begin{array}{c}7.0 \mathrm{~A} \\
\text { Quiescent }\end{array}$ & $23.5 \mathrm{~A}$ \\
\hline $\begin{array}{l}\text { CD-II } \\
\text { Nordan } \\
\text { Goldar } \\
\text { Secar } \\
\text { Bannock } \\
\text { Bozoisky-Select } \\
\text { Magnar } \\
\text { Trailhead }\end{array}$ & $\begin{array}{l}55 \pm 3.5 \mathrm{de} \\
56 \pm 2.4 \mathrm{e} \\
44 \pm 6.1 \mathrm{~cd} \\
23 \pm 3.3 \mathrm{~b} \\
42 \pm 3.4 \mathrm{c} \\
23 \pm 7.2 \mathrm{~b} \\
8 \pm 2.3 \mathrm{a} \\
7 \pm 2.4 \mathrm{a}\end{array}$ & $\begin{array}{c}14.9 \pm 0.5 \mathrm{c} \\
15.2 \pm 1.3 \mathrm{c} \\
12.7 \pm 2.2 \mathrm{bc} \\
4.1 \pm 0.3 \mathrm{a} \\
9.7 \pm 1.1 \mathrm{~b} \\
3.7 \pm 0.9 \mathrm{a} \\
2.2 \pm 0.4 \mathrm{a} \\
2.0 \pm 0.7 \mathrm{a}\end{array}$ & $\begin{array}{l}27.3 \pm 0.8 \mathrm{~cd} \\
26.8 \pm 1.3 \mathrm{~cd} \\
28.4 \pm 1.0 \mathrm{~d} \\
18.3 \pm 1.1 \mathrm{ab} \\
22.9 \pm 1.7 \mathrm{bc} \\
16.9 \pm 1.4 \mathrm{a} \\
27.4 \pm 3.2 \mathrm{~cd} \\
26.7 \pm 1.0 \mathrm{~cd}\end{array}$ \\
\hline Mean & $32 \mathrm{~B}$ & $8.0 \mathrm{~A}$ & $24.3 \mathrm{~A}$ \\
\hline
\end{tabular}

${ }^{1}$ Variety means within a column and stage of phenology sharing a common lower case letter are not significantly different $(P>0.05)$. Means in columns sharing a common upper case letter are not significantly different $(\mathrm{P}>0.05)$.

During boot-stage trials moisture content ranged from $66.7 \%$ for Goldar to $76.3 \%$ for Trailhead and averaged $69.6 \%$. The slightly higher moisture content of Trailhead and Magnar may have been due to their less advanced phenology (Table 2). After grasses were quiescent, moisture content averaged $25 \%$ and ranged between 22.7 (Bannock) to 30.4\% (CD-II). Some have speculated that livestock preferences are positively associated with moisture content of forages (Gesshe and Walton 1980). Our data did not support this hypothesis since 2 of only 3 significant correlations between plant moisture content and our grazing behavior variables were negative in sign.

Forage density is an integration of herbage biomass and basal area; and bulk density is an integration of biomass, basal area, and plant height. In boot-stage trials Bozoisky-Select was lowest in forage density $\left(0.08 \mathrm{~g} / \mathrm{cm}^{2}\right)$ and Magnar and Goldar ranked highest $\left(0.23 \mathrm{~g} / \mathrm{cm}^{2}\right)$. At quiescence the rhizomatous Bannock ranked lowest and Secar and Trailhead were highest (Table 2). No differences occurred among varieties in bulk density in bootstage trials, but differences were significant at quiescence. The rhizomatous Bannock ranked lowest in bulk density at $2.7 \mathrm{~g} / \mathrm{l}$ and Secar was highest at $7.3 \mathrm{~g} / \mathrm{l}$.

In well-controlled experiments, Laca et al. (1992) and Distel et al. (1995) showed that cattle graze most efficiently and expend more time where forage density or bulk density allowed the most rapid intake rate. In boot-stage trials no significant correlations occurred between forage density or bulk density and any of our grazing behavior variables. When forages were dormant, however, 6 weak (mean $r=0.57$ ), but significant correlations $(P<0.01$ ) implied that steers did not seek out the highest densities of for- age. We suggest that many of the foraging optimization processes evident in well-controlled environments where selective opportunities are limited are not as rigidly enforced by grazers when they are offered an abundance of diverse high-quality herbage.

CD-II leaf/stem ratios ranked consistently low at both stages of phenology, and Magnar and Trailhead ranked highest in bootstage trials (Table 2). Bozoisky-Select, Bannock, Magnar and Trailhead had the highest leaf/stem ratios at quiescence. Although stem material has been negatively associated with forage acceptability in other research (Ganskopp et al. 1992), accounting for up to $94 \%$ of animal preference in some instances (Murray 1984), we detected no correlations $(\mathrm{P}>0.01$ ) between leaf/stem ratios and any of our indices of forage selection.

\section{Forage and Diet Nutritional Characteristics}

Nutritional characteristics of the grasses and esophageal samples exhibited declines in forage quality with advancing phenology (Table 4), but selective grazing by steers facilitated harvest of a higher quality diet than expected from standing-crop samples. Boot-stage diets contained more $\mathrm{CP}, \mathrm{ADL}, \mathrm{P}, \mathrm{CA}$, and MG than the standing-crop. Similarly, quiescent diets were higher $(P<0.05)$ in $\mathrm{CP}, \mathrm{ADL}$, and $\mathrm{P}$ than the plants on offer. With the exception of phosphorus, analyses of diet samples suggested forages furnished adequate nutrition for lactating beef cattle in both trials (NRC 1984). Phosphorus is typically deficient in the grasses of our region for most of the year (Raleigh and Lesperance 1972), and all of our varieties were well below NRC (1984) recommended levels for cattle $(0.23 \%)$ after they entered quiescence.

\section{Distances Between Feeding Stations}

Only the phenology effect was significant for distances traveled between feeding stations (boot stage mean $=2.4 \mathrm{~m}$ and quiescent $=1.4 \mathrm{~m}$ ). This suggested steers expended more energy grazing during boot-stage trials and made less effort to graze selectively with advancing phenology, an observation that has been noted elsewhere (Stuth et al. 1987). Given our randomized planting arrangement, 8 varieties, and $0.76-\mathrm{m}$ spacing between plant centers, one might expect steers to find a plant to their liking among the 8 nearest neighbors of each feeding station at a mean distance of $0.92 \mathrm{~m}$. Since they averaged $2.4 \mathrm{~m}$ in boot-stage trials and $1.4 \mathrm{~m}$ at quiescence, we suggest the steers selected among the nearest 48 and 24 neighboring plants, respectively, at these 2 stages of phenology.

Algorithms depicting spatial movement of modeled foragers have ranged from random walks at one extreme to the shortest possible path between stations at the other (Gross et al. 1995). The more traditional approach, however, has been a compromise " $\mathrm{L}$ step look-ahead" strategy (Anderson 1983, Hart and Hanson 1990) in which animals look about and evaluate feeding stations within a traveling-window. Recent experiments by Gross et al. (1995) suggested nearest-neighbor rules applied to bighorn sheep (Ovis canadensis) foraging in a patchy alfalfa (Medicago sativa L.) monoculture. Our findings suggest that a seasonally dynamic traveling-window may be most appropriate to beef cattle in patchy environments where a complex array of forages are available.

\section{Correlations Among Grazing Behavior Variables and Predictions of Preferences}

In these trials we assumed bite-count totals were the best measure of steer diets. Although Ortega et al. (1995) suggested 
Table 4. Nutritional characteristics of forages and diets of steers selectively grazing 8 varieties of grasses at 2 stages of phenology on the Northern Great Basin Experimental Range near Burns, Ore. Units for mineral components are micrograms/gram.

\begin{tabular}{|c|c|c|c|c|c|c|c|c|c|}
\hline & CP\% & IVOMD\% & NDF\% & IADF\% & ADL\% & $\mathbf{P}$ & $\mathbf{K}$ & CA & MG \\
\hline & (\%) & (\%) & $(\%)$ & (\%) & $(\%)$ & $\ldots \ldots$ & $\ldots \ldots \ldots$ (ug & g)-- - & 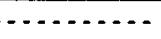 \\
\hline Variety & \multicolumn{9}{|c|}{ Boot Stage } \\
\hline $\begin{array}{l}\text { CD-II } \\
\text { Nordan }\end{array}$ & $\begin{array}{l}20.7 \pm 1.1 \mathrm{a}^{1} \\
20.6 \pm 0.6 \mathrm{a}\end{array}$ & $\begin{array}{l}86.8 \pm 2.3 \mathrm{a} \\
85.2 \pm 1.1 \mathrm{ab}\end{array}$ & $\begin{array}{l}36.2 \pm 0.7 \mathrm{a} \\
37.9 \pm 1.4 \mathrm{ab}\end{array}$ & $\begin{array}{l}6.2 \pm 0.7 a \\
6.3 \pm 0.2 a\end{array}$ & $1.9 \pm 0.1 \mathrm{~b}$ & $2718 \pm 185$ bc & $\begin{array}{l}26014 \pm 782 \mathrm{ab} \\
26636\end{array}$ & $2183 \pm 238 \mathrm{ab}$ & $1163 \pm 34 \mathrm{abcd}$ \\
\hline Goldar & $18.3 \pm 0.5 \mathrm{~b}$ & $81.0 \pm 2.3 \mathrm{ab}$ & $46.0 \pm 3.1 d c$ & $10.8 \pm 0.4 d$ & $2.5 \pm 0.2 \mathrm{~d}$ & $2820 \pm 65 \mathrm{dc}$ & $24446 \pm 95 \mathrm{ab}$ & $1898 \pm 42 a$ & $925 \pm 32 a$ \\
\hline Secar & $20.5 \pm 0.4 a$ & $81.9 \pm 1.8 \mathrm{ab}$ & $47.0 \pm 0.3 d$ & $9.7 \pm 0.8 \mathrm{~cd}$ & $2.0 \pm 0.2 \mathrm{bc}$ & $2945 \pm 127 \mathrm{dc}$ & $26975 \pm 445 \mathrm{ab}$ & $2842 \pm 248 c$ & $1362 \pm 97$ de \\
\hline Bannock & $15.2 \pm 0.3 \mathrm{c}$ & $77.6 \pm 1.7 \mathrm{~b}$ & $51.8 \pm 0.8 \mathrm{e}$ & $9.7 \pm 0.8 \mathrm{~cd}$ & $2.4 \pm 0.2 \mathrm{~cd}$ & $2204 \pm 18 a$ & $21122 \pm 840 \mathrm{a}$ & $2661 \pm 190 b c$ & $1314 \pm 97 \mathrm{cde}$ \\
\hline \multicolumn{10}{|l|}{ Bozoisky- } \\
\hline Select & $19.6 \pm 1.3 \mathrm{ab}$ & $85.4 \pm 2.6 \mathrm{ab}$ & $41.9 \pm 0.8 b c$ & $7.1 \pm 0.6 \mathrm{ab}$ & $1.8 \pm 0.0 \mathrm{ab}$ & $2307 \pm 307 a$ & $34216 \pm 1319 \mathrm{~cd}$ & $2080 \pm 213 \mathrm{ab}$ & $1497 \pm 98$ \\
\hline Magnar & $19.7 \pm 1.2 \mathrm{ab}$ & $85.3 \pm 1.3 \mathrm{ab}$ & $42.8 \pm 0.8 \mathrm{c}$ & $7.5 \pm 0.3 \mathrm{abc}$ & $1.4 \pm 0.3 a$ & $2943 \pm 312 \mathrm{dc}$ & $39956 \pm 1624 d$ & $2100 \pm 118 \mathrm{ab}$ & $1079 \pm 88 \mathrm{abc}$ \\
\hline Trailhead & $18.4 \pm 1.6 \mathrm{~b}$ & $84.0 \pm 2.3 \mathrm{ab}$ & $43.4 \pm 2.8 \mathrm{dc}$ & $8.9 \pm 1.8 \mathrm{bcd}$ & $1.6 \pm 0.3 \mathrm{ab}$ & $2476 \pm 301 \mathrm{ab}$ & $31175 \pm 4237 b c$ & $2118 \pm 284 a b$ & $1022 \pm 71 \mathrm{ab}$ \\
\hline Mean & 19.1 & 83.4 & 43.4 & 8.3 & 1.9 & 2680 & 28818 & 2268 & 1192 \\
\hline Diet & $26.1 \pm 1.0 \mathrm{~B}$ & $82.0 \pm 2.5 \mathrm{~A}$ & $53.8 \pm 4.8 \mathrm{~A}$ & $9.4 \pm 2.0 \mathrm{~A}$ & $2.7 \pm 0.5 B$ & $4080 \pm 356 \mathrm{~B}$ & $30668 \pm 1487 A$ & $2911 \pm 850 \mathrm{~B}$ & $1342 \pm 17 \mathrm{~B}$ \\
\hline \multicolumn{10}{|c|}{ Quiescent } \\
\hline CD-II & \multirow{6}{*}{$\begin{array}{l}8.2 \pm 0.3 \mathrm{abc} \\
9.8 \pm 0.9 \mathrm{a} \\
7.6 \pm 0.7 \mathrm{bcd} \\
8.6 \pm 1.1 \mathrm{ab} \\
6.1 \pm 0.4 \mathrm{~d} \\
9.0 \pm 1.3 \mathrm{ab}\end{array}$} & \multirow{2}{*}{$\begin{array}{l}58.9 \pm 2.0 \mathrm{c} \\
72.4 \pm 7.9 \mathrm{~d}\end{array}$} & \multirow{2}{*}{$\begin{array}{l}65.1 \pm 0.3 \mathrm{a} \\
64.7 \pm 0.3 \mathrm{a}\end{array}$} & \multirow{2}{*}{$\begin{array}{l}19.2 \pm 0.7 \mathrm{a} \\
20.2 \pm 1.2 \mathrm{ab}\end{array}$} & \multirow{2}{*}{$\begin{array}{l}4.9 \pm 0.2 \mathrm{a} \\
5.0 \pm 0.2 \mathrm{a}\end{array}$} & $1010 \pm 98 b c$ & \multirow{2}{*}{$\begin{array}{l}11948 \pm 1356 a \\
14398 \pm 1807 a\end{array}$} & \multirow{2}{*}{$\begin{array}{l}2262 \pm 270 \mathrm{ab} \\
2059 \pm 281 \mathrm{a}\end{array}$} & \multirow{2}{*}{$\begin{array}{c}1057 \pm 43 \mathrm{ab} \\
988 \pm 59 \mathrm{a}\end{array}$} \\
\hline Nordan & & & & & & $1097 \pm 56 \mathrm{dc}$ & & & \\
\hline Goldar & & $62.4 \pm 6.6 \mathrm{c}$ & $65.4 \pm 1.6 \mathrm{ab}$ & $20.9 \pm 0.7 \mathrm{abc}$ & $5.6 \pm 0.6 \mathrm{~b}$ & $1078 \pm 81 \mathrm{dc}$ & $10996 \pm 1863 a$ & $3096 \pm 168 \mathrm{~d}$ & $1243 \pm 84 b c$ \\
\hline Secar & & \multirow{2}{*}{$\begin{array}{l}58.3 \pm 4.1 \mathrm{bc} \\
49.7 \pm 1.9 \mathrm{ab}\end{array}$} & \multirow{2}{*}{$\begin{array}{l}69.4 \pm 0.1 b c \\
72.1 \pm 0.1 \quad c\end{array}$} & \multirow{2}{*}{$\begin{array}{l}24.3 \pm 1.4 \mathrm{~d} \\
20.9 \pm 0.7 \mathrm{abc}\end{array}$} & \multirow{2}{*}{$\begin{array}{l}6.0 \pm 0.2 b \\
5.9 \pm 0.2 b\end{array}$} & $1311 \pm 157 \mathrm{~d}$ & \multirow{2}{*}{$\begin{array}{l}8763 \pm 1033 a \\
8730 \pm 135 a\end{array}$} & \multirow{2}{*}{$\begin{array}{l}3148 \pm 501 \mathrm{~d} \\
2290 \pm 1 \mathrm{abc}\end{array}$} & \multirow{2}{*}{$\begin{array}{c}1319 \pm 107 \mathrm{c} \\
955 \pm 41 \mathrm{a}\end{array}$} \\
\hline Bannock & & & & & & $733 \pm 39 \mathrm{ab}$ & & & \\
\hline $\begin{array}{l}\text { Bozoisky- } \\
\text { Select }\end{array}$ & & $54.2 \pm 1.1 \mathrm{abc}$ & $68.2 \pm 2.4 \mathrm{abc}$ & $22.7 \pm 0.8 \mathrm{~cd}$ & $5.9 \pm 0.4 \mathrm{~b}$ & $793 \pm 140 \mathrm{ab}$ & $22737 \pm 1758 \mathrm{~b}$ & $2672 \pm 357 \mathrm{ab}$ & $1683 \pm 147 \mathrm{~d}$ \\
\hline Magnar & $5.8 \pm 0.6 \mathrm{~d}$ & $49.5 \pm 3.6 a$ & $70.0 \pm 1.3 \mathrm{c}$ & $22.4 \pm 0.7 \mathrm{bcd}$ & $4.8 \pm 0.2 a$ & $549 \pm 14 a$ & $13119+2044 a$ & $2890 \pm 251 \mathrm{bcd}$ & $1349 \pm 80 \mathrm{c}$ \\
\hline Trailhead & $6.6 \pm 1.4 \mathrm{~cd}$ & $56.0+3.9 \mathrm{abc}$ & $70.0 \pm 0.5 \mathrm{c}$ & $23.9 \pm 0.7 \mathrm{~d}$ & $4.9 \pm 0.4 \mathrm{a}$ & $573 \pm 73 a$ & $9047 \pm 1412 a$ & $3450 \pm 483 d$ & $1677 \pm 185 \mathrm{~d}$ \\
\hline Mean & $7.7 \quad \mathrm{C}$ & 57.7 & $68.1 \quad \mathrm{~B}$ & $21.8 \quad B$ & $5.4 \quad \mathrm{C}$ & $893 \mathrm{C}$ & 12467 & 2733 A & $1284 \quad A$ \\
\hline Diet & $10.9 \pm 0.8 \mathrm{D}$ & $60.0 \pm 1.9 \mathrm{~B}$ & $68.1 \pm 4.7 \mathrm{~B}$ & $21.7 \pm 0.4 \mathrm{~B}$ & $7.2 \pm 0.2 \mathrm{D}$ & $2193 \pm 135 D$ & $10547 \pm 1378 B$ & $3248 \pm 183 \mathrm{~A}$ & $1039 \pm 77 \mathrm{~A}$ \\
\hline
\end{tabular}

${ }^{\mathrm{T}}$ Variety means within a column and stage of phenology sharing a common lower case letter are not significantly different $(\mathrm{P}>0.05)$.

Means in columns sharing a common upper case letter are not significantly different $(P>0.05)$.

esophageal techniques are superior in dense and structurally diverse environments for quantifying diets, our limited selection of forages and planting arrangement probably allowed an accurate assessment of relative intake among varieties. We found total time expended on each variety was the only variable consistently correlated with total bites per variety in both trials $(r=0.99$ and 0.97 , respectively, during boot and quiescent phenologies). Again, this suggested that measures of time spent with each variety would provide adequate rankings of forages in similar trials if favored plants are not entirely depleted. Acquisition of bite-count data is quite demanding, and others have found that indices of relative time expended with forages may provide adequate interpretations regarding relative preferences (Petersen et al. 1958). Intake may be poorly correlated with grazing time, however, if animals forage among a complex variety of life forms (Stuth et al. 1987).

In boot-stage trials other strong correlations included: visits with number of plants grazed $(r=0.98)$, number of plants regrazed $(r=0.95)$, and the utilization index of number of plants grazed $(r=0.95)$; bites/visit with time/visit $(r=0.98)$; and the utilization count of number of plants grazed with the observed number of plants grazed $(r=0.96)$, and \% weight utilized $(r=0.96)$. In quiescent trials, visits $(r=0.99)$ and total bites $(r=0.95)$ were strongly correlated with the number of plants grazed. All of the above relationships were significant at $P<0.000$.

Efforts to find consistent relationships among the physical and chemical characteristics of varieties and the selective patterns of steers as indexed by total bites were largely unsuccessful. For boot-stage trials our step-wise regression model was:

Total bites $=15.8$ (Plant biomass) +18.4 (IVOMD) $+0.23(\mathrm{P})-0.02(\mathrm{~K})-1324$ with variables listed in their respective order of entry $\left(R^{2}=0.80\right)$.
The non-significant variable entering when analysis was terminated was leaf/stem ratio $(P=0.18)$. For quiescent trials the model was:

Total bites $=-24.8(\mathrm{IADF})-0.52(\mathrm{CA})+0.48(\mathrm{P})-757.5$

with variables again listed in respective order of entry $\left(R^{2}=0.72\right)$. Again leaf/stem ratio was the first non-significant variable to enter the model $(P=0.07)$. Phosphorus was the only variable consistently present in both models, and on entry it contributed 0.25 and 0.10 , respectively, to boot stage and quiescent coefficients of determination.

\section{Conclusions}

Steers foraged most selectively among our 8 varieties during boot-stage trials, when the CD-II and Nordan crested wheatgrasses were preferred. Those 2 varieties collectively accounted for $53 \%$ of total bites harvested by steers. Our findings based on total bites, total time, and plants regrazed also suggested CD-II was favored over Nordan crested wheatgrass. At quiescence Bannock was preferred over the closely related Secar based on bites per visit, percentages of single bite visits, time/visit, and bite rate differences. No differences in preference occurred between the 2 Basin wildrye varieties (Magnar and Trailhead).

The degree of selectivity exhibited in boot-stage trials emphasizes the need to be cognizant of livestock preferences in pastures of mixed composition. Preferred forages have a much higher probability of being grazed, have more bites removed during each visit, and preferred plants are regrazed well before any of the forages are depleted. This focus on preferred grasses during the boot 
stage of phenology may adversely affect palatable species over the long run, and ungrazed forages may eventually gain a competitive advantage in a pasture. Conversely, since steers were less selective and grasses are least affected by defoliation at quiescence, pasture clean-up may be more easily accomplished at this later stage of phenology.

Recent interest in optimum foraging theory, inexpensive computer access, and desires to develop accurate predictive tools, have stimulated development of models mimicing the temporal, spatial, quantitative, and qualitative aspects of herbivore foraging. Our efforts to detect variables that may consistently predict forage selection by steers were largely unsuccessful. Our spatial observations suggest, however, that a seasonally dynamic moving-window is an appropriate algorithm for simulating beef cattle foraging in a mixed-composition, patchy environment. Boot stage and quiescent travel distances between feeding stations averaged 2.4 and $1.4 \mathrm{~m}$, respectively, implying that steers selected from among the nearest 48 and 24 neighbors at these 2 stages of phenology. On a similar note, steers grazed the nearest available plant $50 \%$ of the time in boot-stage trials and $65 \%$ of the time when forages were dormant. We still have much to learn before we can create realistic models of foraging behavior, and a coordinated effort by many disciplines will be required to successfully complete this task.

In reference to the several indices of grazing behavior and preference used in this study, we suggest that the more easily acquired measure of relative grazing time is adequate for ranking preferences of steers. Acquisition of bite-count data in the field is demanding, and others have found that indices of relative time expended with forages typically provide adequate interpretations. Exceptions to this occur, however, when animals forage among a variety of life forms where intake may be poorly correlated with grazing time. Although rankings of forages based on post grazing counts of plants utilized and percent of plant weight utilized closely corresponded with our bite count data (mean $r_{s}=0.90$ ), we agree with others that utilization methods are not a preferred means of quantifying herbivore diets.

All varieties evaluated in these trials provided suitable forage for cattle through the growing season and into early dormancy. All were deficient in phosphorus as the growing season progressed, so cattle should receive some supplement. Each variety is ideally suited to specific soil, moisture, and climatic regimes, and each will be grazed if cattle have a limited selective opportunity. The proven ease of establishment, competitive ability, nutritional value, grazing tolerance, and relative palatabilities of the 2 crested wheatgrass cultivars (CD-II and Nordan) suggests both are excellent candidates for pasture reclamation or establishment in beef production programs. Although forage management becomes more complex in pastures of mixed composition, less palatable cultivars might be used in critical or sensitive areas to discourage livestock grazing or enhance the preservation of ground cover.

\section{Literature Cited}

Anderson, D.J. 1983. Optimal foraging and the traveling salesman. Theor. Pop. Biol. 24:145-159.

Anderson, E.W. and R.J. Scherzinger. 1975. Improving quality of winter forage for elk by cattle grazing. J. Range Manage. 28:120-125.

AOAC. 1980. Official methods of analysis of the Association of Official Analytical Chemists. Washington, D.C., 1018 pp.
Brown, J.R. and J.W. Stuth. 1993. How herbivory affects grazing tolerant and sensitive grasses in a central Texas grassland: integrating plant response across hierarchical levels. Oikos 67:291-298.

Crawley, M.J. 1990. Rabbit grazing, plant competition and seedling recruitment in arid grassland. J. Appl. Ecol. 27:803-820.

Distel, R.A., R.A. Laca, T.C. Griggs, and M.W. Demment. 1995. Patch selection by cattle: maximization of intake rate in horizontally heterogeneous pastures. Appl. Anim. Behav. Sci. 45:11-21.

Fajemisin, B., D. Ganskopp, R. Cruz, and M. Vavra. 1996. Potential for woody plant control by Spanish goats in the sagebrush steppe. Small Rumin. Res. 20:99-107.

Galyean, M.L., L.J. Krysl, and R.E. Estell. 1987. Marker-based approaches for estimation of fecal output and digestibility in ruminants. In: F.N. Owens (ed.) Feed intake by beef cattle: Symp. Oklahoma Agr. Exp. Sta. MP-121:96

Ganskopp, D., R. Angell, and J. Rose. 1992. Response of cattle to cured reproductive stems in a caespitose grass. J. Range Manage. 45:401-404.

Ganskopp, D., B. Myers, and S. Lambert. 1993. Black-tailed jackrabbit preferences for eight forages used for reclamation of Great Basin rangelands. Northwest Sci. 67:246-250.

Ganskopp, D., L. Richman, D. Johnson, R. Angell, and R. Cruz. 1996. Preferences of Angora goats for eight selections of grasses used for reclamation of Great Basin rangelands. Small Rumin. Res. 19:103-112.

Gesshe, R.H. and P.D. Walton. 1980. Forage preferences. Alberta Agr. Forest. Bull. 3:10-13.

Goering, H.K. and P.J. Van Soest. 1970. Forage fiber analysis (apparatus, reagents, procedures, and some applications). Agr. Handb. No. 379. ARS, USDA. Washington, D.C.

Gordon, I.J. 1988. Facilitation of red deer grazing by cattle and its impact on red deer performance. J. App. Ecol. 25-1-10.

Gross, J.E., C. Zank, N.T. Hobbs, and D.E. Spalinger. 1995. Movement rules for herbivores in spatially heterogeneous environments: responses to small scale pattern. Landscape Ecol. 10:209-217.

Hart, R.H. and J.D. Hanson. 1990. PASTORAL grazing simulator. J. Agron. Educ. 19:55-58.

Jones, T.A., M.H. Ralphs, and D.C. Nielson. 1994. Cattle preference for 4 wheatgrass taxa. J. Range Manage. 47:119-122.

Laca, E.A., D. Ungar, N. Seligman, and M.W. Demment. 1992. Effects of sward height and bulk density on bite dimensions of cattle grazing homogeneous swards. Grass Forest. Sci. 47:91-102.

Laycock, W.A., H. Buchanan, and W.C. Krueger. 1972. Three methods of determining diet, utilization, and trampling damage on sheep range. J. Range Manage. 25:352-356.

Lentz, R.D. and G.H. Simonson. 1986. A detailed soils inventory and asociated vegetation of Squaw Butte Range Experiment Station. Agr. Exp. Sta. Oregon State Univ., Corvallis. Special Rep. 760.

McInnis, M.L., M. Vavra, and W.C. Krueger. 1983. A comparison of four methods used to determine the diets of large herbivores. J. Range Manage. 36:302-306.

McNaughton, S.J. and N.J. Georgiadis. 1986. Ecology of African grazing and browsing mammals. Annu. Rev. Ecol. Syst. 17:39-65.

Murray, R.B. 1984. Yield, nutrient quality, and palatability to sheep of fourteen grass accessions for potential use on sagebrush-grass range in southeastern Idaho. J. Range Manage. 37:343-348.

NRC (National Research Council)-National Academy Press. 1984. Nutrient requirements of beef cattle. 6th ed., Washington, D.C. 90 pp.

Ortega, I.M., F.C. Bryant, and D.L. Drawe. 1995 Contrast of esophageal-fistula versus bite-count techniques to determine cattle diets. J. Range Manage. 48:498-502.

Petersen, R.G., P.H Wesig, and J. R. Cowan. 1958. Measuring palatability differences in tall fescue by grazing sheep. Agron. J. 50:117-119.

Raleigh, R.J. and A.L. Lesperance. 1972. Range cattle nutrition. p. 185-199d. In: D.C. Church (ed.) Digestive physiology and nutrition of ruminants. Dept. Anim. Sci. Oregon State Univ., Corvallis, Ore.

Reiner, R.J. and P.J. Urness. 1982. Effect of grazing horses managed as manipulators of big game winter range. J. Range Manage. 35:567-571. 
Sharrow, S.H. and W.D. Mosher. 1982. Sheep as a biological control agent for tansy ragweed. J. Range Manage. 35:480-482.

Sharrow, S.H., W.C. Leininger, and B. Rhodes. 1989. Sheep grazing as a silvicultural tool to suppress brush. J. Range Manage. 42:2-4.

Shewmaker, G.E., H.F. Mayland, R.C. Rosenau, and K.H. Asay. 1989. Silicon in C-3 grasses: effects on forage quality and sheep preference. J. Range Manage. 42:122-127.

Sneva, F.A. 1982. Relation of precipitation and temperature with yield of herbaceous plants in eastern Oregon. Int. J. Biometeor. 26:263-276.

Stuth, J.W., J.R. Brown, P.D. Olson, M.R. Araujo, and H.D. Aljoe. 1987. Effects of stocking rate on critical plant-animal interactions in a rotational grazed Schizachyrium-Paspalum savanna. p. 115-139, In:
F.P. Horn, J. Hodgson, J.J. Mott and R.N. Brougham (eds.), Grazinglands research at the plant-animal interface. Winrock Int., Morrilton, Ark.. USA.

Taylor, C.A., T.D. Brooks, and N.E. Garza. 1993. Effects of short duration and high-intensity, low-frequency grazing systems on forage production and composition. J. Range Manage. 46:118-121.

Tilley, J.M.A. and R.A. Terry. 1963. A two-stage technique for the invitro digestion of forage crops. J. Brit. Grassl. Soc. 18:104-111.

Walker, J.W., K.G. Hemenway, P.G. Hatfield, and H.A. Glimp. 1992. Training lambs to be weed eaters: studies with leafy spurge. J. Range Manage. 45:245-249.

\section{Just published- \\ Ecology and Management \\ of North American Savannas}

\section{GUY R. MCPHERSON}

Because savannas are generally thought of as tropical ecosystems, most reviews of the literature have tended to disregard those found in temperate zones. Yet these ecosystems are both extensive and diverse in North America, ranging from longleaf pine habitats along the Atlantic coastal plain to xeric pinon-juniper communities of the Great Basin.

This book provides an overview of the patterns and processes shared by these ecosystems and offers substantive ideas regarding future management and research efforts. It describes the composition, geographic distribution, climate, soils, and uses of savannas throughout North America, summarizing and integrating a wide array of literature. While discussing these ecological patterns and processes, McPherson develops a framework for implementing management practices and safeguarding the future of these important wildland ecosystems.

224 pp., 29 illus. ISBN 0-8165-1624-3. \$35.00

Now in paperback-

\section{The Desert Grassland}

\section{Edited by MITCHEL P. McCLARAN and THOMAS R. VAN DEVENDER}

"An integrated, comprehensive, and thought-provoking overview." - Choice "Students, managers and scientists should all find The Desert Grassland useful and interesting." -Journal of Range Management

346 pp., 109 illus. ISBN 0-8165-1823-8. \$19.95

\section{The University of Arizona Press}

1230 N. Park Ave., Tucson AZ $85719 \bullet$ 1-800-426-3797 • http://www.uapress.arizona.edu 